\title{
SHORTER COMMUNICATIONS
}

\section{THE EFFECTS OF AXIAL HEAT CONDUCTION ON THE FREEZING OR MELTING OF CYLINDERS}

\author{
GEORGE S. SPRINGER
}

Fluid Dynamics Laboratory, Department of Mechanical Engineering, The University of Michigan, Ann Arbor, Michigan

(Received 29 July 1968 and in revised form 24 October 1968)

\section{NOMENCLATURE}

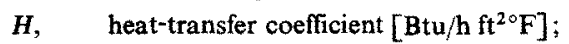

$H^{*}$, dimensionless heat-transfer coefficient, $=\mathrm{Hr}_{i} / k_{s}$;

$k$, thermal conductivity [Btu $\left./ \mathrm{hft}^{\circ} \mathrm{F}\right]$;

$K, \quad$ conductivity ratio, $=k_{\mathrm{L}} / k_{s}$;

$Q^{*}, \quad$ dimensionless heat flux, $=Q / 2 \pi k_{S} T_{F}$;

$r, z, \quad$ radial and axial coordinates $[\mathrm{ft}]$;

$R, \quad$ outer radius [ $\mathrm{ft}]$;

$r^{*}, z^{*}$, dimensionless radial and axial coordinates, $=$ $\left(r-r_{i}\right) / r_{i}$ and $z / r_{i}$;

$S$, length of cylinder [ft $]$;

$T, \quad$ temperature $\left[{ }^{\circ} \mathrm{F}\right]$;

$t, \quad$ time [hr];

$t^{*}, \quad$ dimensionless time, $=T_{F} k_{S} t / \rho L r_{i}^{2}$;

$\beta, \quad$ material property, $=L / c T_{F}$;

$\xi$ fusion front position [ft] (see Fig. 1):

$\xi^{*}, \quad$ dimensionless fusion front position $=\left(\xi-r_{1}\right) / r_{i}$;

$\phi, \quad$ fusion front slope, [rad.] (see Fig. 1);

$\theta, \quad$ dimensionless temperature, $\left(T-T_{F}\right) / T_{F}$.

\section{Subscripts}

$F$, fusion condition;

$i, \quad$ inner radial boundary;

$L$, liquid phase;

0 , initial temperature ;

$S, \quad$ solid phase;

$\infty$ ambient condition.

\section{INTRODUCTION}

Most of the analyses available for describing the freezing or melting of cylinders [1-10] are based on the assumption that heat transfer and temperature variations in the axial direction are negligibie. The validity of this assumption must be known, however, before applying the results of such simplified calculations to practical problems. Therefore, an attempt is made here to establish the conditions under which the heat transfer rates and the fusion front motions may be calculated with reasonable accuracy by neglecting all axial gradients.
Due to the complexity of the problem numerical solutions are obtained only for the problem where the material undergoing the phase change is contained between two concentric cylinders of finite radius ratio and of finite length. Solutions to such problems are of interest in heat exchangers and in heat storage systems. Although such applications are of prime concern in this analysis, the results are sufficiently general so as to be useful in other problems of similar geometry.

\section{THE PROBLEM}

A given material with a distinct fusion temperature, $T_{F}$, is contained between two concentric cylinders (Fig. 1). Initially $(t<0)$ only one phase is present and its temperature $T_{0}$ is constant. At $t \geqslant 0$ a known temperature distribution, $T_{\infty}(z)$, is imposed along the inner cylindrical surface. $T_{\omega}$ may vary along $z$ but is independent of the angular coordinate and time. Heat is transferred in or out of the material through the inner radial surface, resulting in melting or freezing. The fusion front moves towards the outer radius. The outer cylindrical surface and the ends of the cylinder are assumed to be adiabatic. The problem is to determine the heat transfer rates and the positions of the fusion front as functions of time. For homogeneous materials with constant thermal properties and for the assumption that heat is transferred through the material by conduction only, Fourier's equation for the liquid and solid phases gives

$$
\begin{aligned}
& \frac{\partial T_{L}}{\partial t}=\propto_{L}\left(\frac{\partial^{2} T_{L}}{\partial r^{2}}+\frac{1}{r} \frac{\partial T_{L}}{\partial r}+\frac{\partial^{2} T_{L}}{\partial z^{2}}\right) \\
& \frac{\partial T_{S}}{\partial t}=\propto_{S}\left(\frac{\partial^{2} T_{S}}{\partial r^{2}}+\frac{1}{r} \frac{\partial T_{S}}{\partial r}+\frac{\partial^{2} T_{S}}{\partial z^{2}}\right) .
\end{aligned}
$$

By taking the densities of the two phases to be equal $\left(\rho_{L}=\rho_{S}=\rho\right)$ a heat balance at the interphase yields

$$
\left\{ \pm \rho L \frac{\partial \xi}{\partial t}=\left(k_{s} \frac{\partial T_{s}}{\partial r}-k_{L} \frac{\partial T_{L}}{\partial r}\right)\left(1+\tan ^{2} \phi\right)\right\}_{r \approx \xi} .
$$




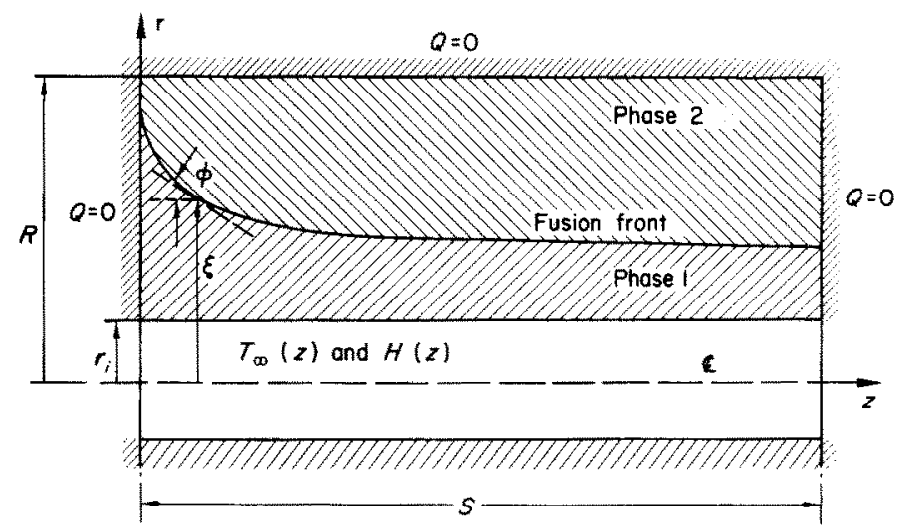

FIG. 1. Physical model used in the analysis.

In equations (1) and (2) $\propto$ is the thermal diffusivity, $L$ the latent heat of fusion. All other symbols are defined in the nomenclature. The positive and negative signs are for melting and freezing respectively. The initial and boundary conditions are:

$$
\begin{aligned}
& t<0 \\
& \quad T=T_{S}=T_{L}=T_{0} \text { and } \xi=0 \\
& \quad \text { at } r_{i} \leqslant r \leqslant R \text { and } 0 \leqslant z \leqslant S
\end{aligned}
$$

$1 \geqslant 0$

$$
\begin{aligned}
T=T_{\infty}(z) \text { and } H=H(z) \\
\qquad \text { at } r=r_{1} \text { and } 0 \leqslant z \leqslant S
\end{aligned}
$$

$\hat{\partial T / \partial r}=0 \quad$ at $r=R$ and $0 \leqslant z \leqslant S$

$\partial T / \partial z=0 \quad$ at $z=0$ and $z=S$

$$
\text { and } r_{i} \leqslant r \leqslant R
$$

Denoting by $\Delta T$ the temperature difference between $T_{\infty}$ and the temperature of the medium at $r=r_{i}$ the heat flux per unit length through the inner boundary is $Q=2 \pi r_{i} H \Delta T$.

Equations (1) $(3)$ were transformed into dimensionless form using the parameters $r^{*}, \xi^{*}, z^{*}, S^{*}, t^{*}, \theta, \beta, K, H^{*}$ and $Q^{*}$ as defined in the nomenclature. The eight parameters that must be specified prior to the solution are $\beta_{S}, \beta_{L}, K, \theta_{0}$, $\theta_{\infty}, H^{*}, R^{*}$ and $S^{*}$. It is important to choose for the calculations those values of these parameters which include conditions likely to occur in practice. For most common materials $\beta_{S}$ and $\beta_{L}$ range from 0.5 to 20 , and $K$ from 0.5 to 10 [10], with the exception of water, for which the average values are $\beta_{S} \cong 9.5, \beta_{L} \cong 4.5$ and $K \cong 0.3$. The initial temperature of the liquid may lie between the fusion and the boiling temperatures. The ambient temperature along the boundary must be less than the bolling temperature during melting. In freezing processes ambient temperatures are not likely to exceed the value of -1.0 except for ice formation, where it may be lower. Along the inner boundary $H^{*}$ is expected to be in the range $10-50$. In most applications the minimum value of $r_{0}^{*}$ is approximately 2:1 while the lower limit for $S^{*}$ is about 10:1. Solutions have been obtained for parameters selected from the ranges indicated above using the numerical techniques described in [11].

\section{RESULTS}

The problems described by equations (1)-(3) may be divided into two groups: (1) the axial temperature gradients and the fusion front slope are zero at all points inside the material $\partial T / \partial z=0$ and $\phi=0$ ), and all parameters depend only on $r$, and (2) $\partial T / \partial z \neq 0$ and $\phi \neq 0$. Here problems in the second group will be investigated. The aim is to determine the conditions under which axial heat transfer effects are insignificant, because the results of simpler calculations performed for $\partial T / \partial z=0, \phi=0$ could then be applied to the more difficult problems where $\partial T / \partial z \neq 0, \phi \neq 0$. Furthermore, an attempt is made to establish simulated boundary conditions which (when used in place of the actual boundary conditions) would permit the use of the simpler analyses (group (1) above) for calculating heattransfer rates and fusion front positions in the more complex three dimensional problems (group (2)).

In order to establish a temperature gradient in the material an ambient temperature varying experimentally with $z^{*}$ is imposed along $r_{i^{*}}$ Such a temperature variation may exist for instance when a secondary fluid flows in the axial direction adjacent to the boundary [12]. Using the freezing process as a sample and allowing a variation in the cooling temperatures from $\theta=1.0\left(\right.$ at $z^{*}=0$ ) to $\theta=-0.25$ (at $\left.z^{*}=S^{*}\right), \theta_{\infty}=-1.0 \exp \left(-1.34 z^{*} / S^{*}\right)$. For this ambient temperature the effects of $r_{0}^{*}$ and $S^{*}, \beta_{S}, \beta_{L}$ and $K$ on the axial gradients can now be evaluated First, the fusion front slope was calculated, since $\phi$ depends strongly on the axial heat conduction. The maximum values of $\phi$ occur at $z^{*}=0$, for the above $\theta_{\infty}$. Thus $\phi$ was computed at $z^{*}=0$ at various 


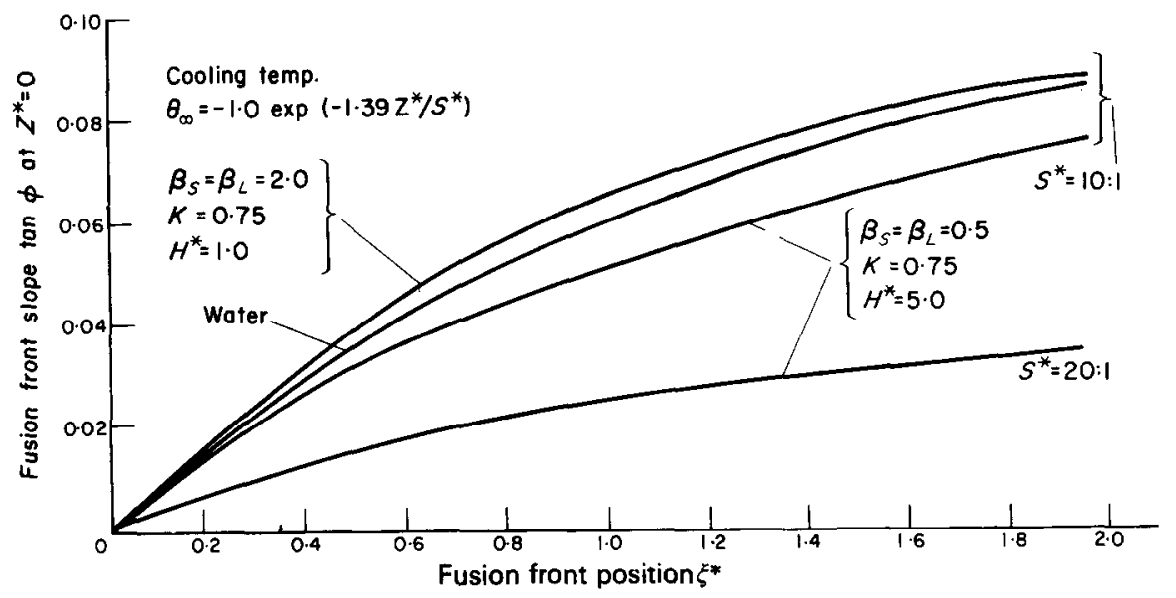

FIG. 2. The maximum fusion front slopes during a freezing process for $\theta_{0}=0, r_{0}^{*}=2: 1$.

fusion front positions and for various $S^{*}, \beta_{S}, \beta_{L}$ and $K$ values, for $r_{0}^{*}=2: 1$ and $\theta_{0}=0 \cdot 0$. From Fig. 2 it can be seen that as the fusion front moves towards the outer radial boundary $\phi$ increases. However, even for the extreme values of the parameters used in the calculations the slope is small $\left(\phi \cong 0-6^{\circ}\right)$. The maximum slope and consequently the maximum axial heat conduction effects occur when $\beta_{S}=$ $\beta_{L}=2 \cdot 0, K=0.75$, and $H^{*}=1 \cdot 0$, and these values will be used in the further computations. Note that the fusion tront slope is rather insensitive to the material properties, but strongly depends on the length. When $S^{*}$ is increased from $10: 1$ to $20: 1$, the maximum slope is reduced by about a factor of two (Fig. 2).

The calculated fusion front positions $\xi^{*}$ at $z^{*}=0$ and at $z^{*}=S^{*}$ are shown in Fig. 3 as a function of time. $\xi^{*}$ was also calculated at these same two axial positions by neglecting axial temperature gradients and by taking the ambient temperatures to be equal to those that exist at these positions, (i.e. at $z^{*}=0, \theta_{\infty}=-1.0$ and at $z^{*}=S^{*}, \theta_{\infty}=$ -0.25 ). The results for the complete and approximate analyses agree well. Thus, the fusion front position may be calculated with reasonable accuracy from appropriate two dimensional analyses by first breaking up the cylinder into suitably small elements and then replacing the axially varying ambient temperature distribution with the average value of $\theta_{\infty}$ that exists at the boundary surface of this element.

In Fig. 4 is presented the heat transfer through the inner boundary of the cylinder, when $\theta_{\infty}$ varies along $z^{*}$. By dividing into two equal parts the arc length of the exponential curve connecting $\theta_{\infty}$ at $z^{*}=0$ and $\theta_{\infty}$ at $z^{*}=S^{*}$ a constant "equivalent temperature" has also been obtained which, when applied uniformly along $r_{i}$, yields the same heat transfer rate as the axially varying $\theta_{\infty}$. The results computed with these temperatures are in good agreement with those

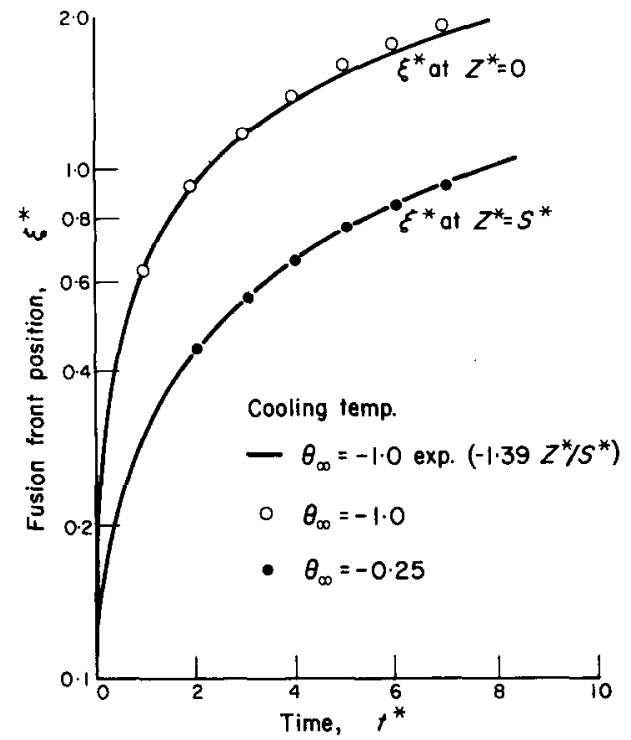

Fig. 3. Fusion front positions calculated for axially varying ambient temperature distribution (solid lines) and for constant ambient temperatures (circles). $\left(\beta_{S}=\beta_{L}=2 \cdot 0, \quad K=0.75, \quad H^{*}=1.0, \quad S^{*}=10: 1\right.$, $\left.r_{0}^{*}=2: 1, \theta_{0}=0\right)$.

calculated from the nonuniform axial temperature distribution.

It may be concluded now that in many cylindrical freezing and melting problems of practical interest the heat transfer and fusion front positions can be calculated by neglecting the axial heat conduction and temperature gradients, provided that appropriate boundary conditions are used. Suitable approximate solutions obtained by neglecting 


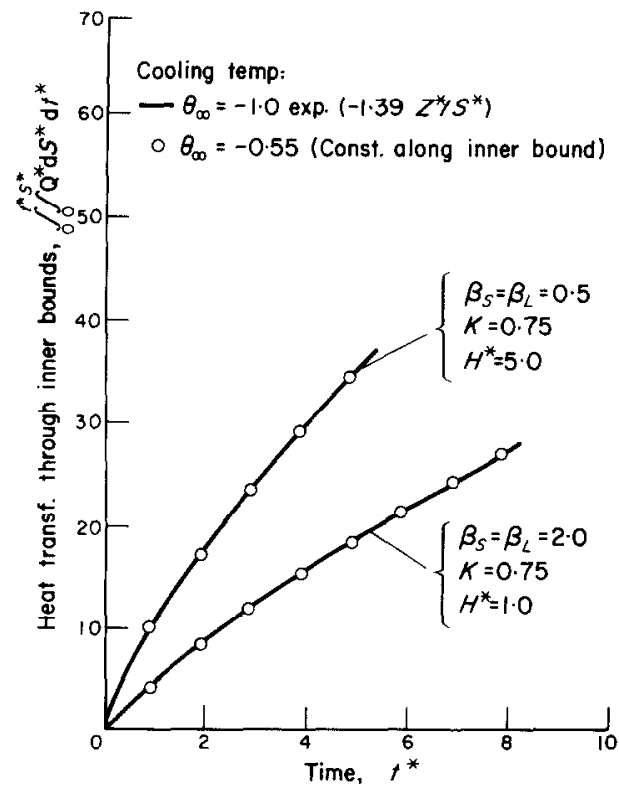

Fig. 4. Heat transter through inner boundary surface of the cylinder calculated for axially varying temperature distribution (solid lines) and for constant "equivalent" ambient temperatures (circles). $\left(S^{*}=10: 1, r_{0}^{*}=2: 1, \theta_{0}=0\right)$.

axial heat-transfer effects for various initial and bonndary conditions are given in [1-3] and [6-10].

\section{ACKNOWLEDGEMENTS}

This work was supported in part by the National Science Foundation under Grant No. GK-1745.

\section{REFERENCES}

1. C. L. Pekeris and L. B. Slichter, Problem of ice formation, J. Appl. Phys. 10, 135 (1939).

2. A. L. LONDON and R. A. SEBAN, Rate of ice-formation, Trans. Am. Soc. Mech. Engrs 65, 771 (1943).

3. L. R. Ingersoll, F. T. Adler, H. J. Plass and A. C. INGERSOLL, Theory of earth heat exchangers for the heat pump, Heat. Pip. Air Condit. 22, 113 (1950).

4. L. I. Rubinstein, Heat transfer in a two-phase medium in the case of cylindrical symmetry, Dokl. Akad. Nauk. SSSR. 79, 945 (1951).

5. A. B. Decev, On the two-dimensional Stefan's problem, Dokl. Akad. Nauk. SSSR. 101, 441 (1955).

6. F. KReIri and F. E. RomIE, A study of the thermal diffusion equation with boundary conditions corresponding to solidification or melting of materials initially at fusion temperature, Proc. Phys. Soc., London 68, (B), 283 (1955).

7. H. S. Carslaw and J. C. JAEGer, Conduction of Heat in Solids, p. 296. Oxford University Press, London (1959).

8. C. F. Dewey, S. I. Schlesinger and L Sashkin, Temperature profiles in a finite solid with moving boundary, J. Aero/Space Sci. 27, 59 (1960).

9. D. C. BAXTER, The fusion times of slabs and cylinders, ASME Paper No. 61-WA-179 (1961).

10. G. S. Springer and D. R. Olson, Axisymmetric solidification and melting of materials, ASME Paper No. 63-WA-185 (1963).

11. G. S. SPringer and D. R. Olson, Method of solution of axisymmetric solidification and melting problems, ASME Paper No. 62-WA-242 (1962).

12. M. JACOB, Heat Transfer, Vol. 2, p. 208. John Wiley, New York (1957).

\title{
HEAT FLOW ACROSS METALLIC JOINTS- THE CONSTRICTION ALLEVIATION FACTOR
}

\author{
A. HUNTER and A. WILLIAMS \\ Mechanical Engineering Department, Monash University, Clayton, Victoria, Australia
}

(Received 23 Sebtember 1968 and in revised form 7 November 1968)

\section{NOMENCLATURE}

$\Gamma, \quad$ temperature;

$Q$, total heat flow;

$\Delta T$, temperature drop due to one side of constriction (see Fig. 1(a));

$R$, thermal resistance of one side of constriction $\Delta T / Q$;

$k$, thermal conductivity;

a, radius of metallic contaet spot (see Fig 1(b));

$b$, radius of cylindrical region feeding the contact; $f$ constriction alleviation factor, defined as the ratio of the actual resistance to that of the disc constriction resistance when bounded by a semi infinite conductor;

$u, t$, dummy variables of integration.

\section{INTRODUCTION}

IN THE study of the thermal resistance of metallic contacts it is usual to model the conductors as a series of cylindrical 